(2) Open Access Full Text Article

REVIEW

\title{
Assessing quality of life in the treatment of patients with age-related macular degeneration: clinical research findings and recommendations for clinical practice
}

\author{
This article was published in the following Dove Press journal: \\ Clinical Ophthalmology \\ 28 June 2013 \\ Number of times this article has been viewed
}

\author{
Mitsuko Yuzawa' \\ Kyoko Fujita' \\ Erika Tanaka ${ }^{2}$ \\ Edward CY Wang ${ }^{2}$ \\ 'Department of Ophthalmology, \\ Nihon University School of Medicine, \\ Surugadai, Kanda, Chiyoda-ku, \\ Tokyo, Japan; ${ }^{2}$ Health Economics \\ and Outcomes Research, Bayer \\ Yakuhin Ltd, Marunouchi, Chiyoda-ku, \\ Tokyo, Japan
}

\begin{abstract}
Background: The importance of incorporating quality-of-life (QoL) assessments into medical practice is growing as health care practice shifts from a "disease-based" to a "patient-centered" model. The prevalence of age-related macular degeneration (AMD) is increasing in today's aging population. The purpose of this paper is: (1) to discuss, by reviewing the current literature, the impact of AMD on patients' QoL and the utility of QoL assessments in evaluating the impact of AMD and its treatment; and (2) to make a recommendation for incorporating QoL into clinical practice.
\end{abstract}

Methods: We conducted a PubMed and an open Internet search to identify publications on the measurement of QoL in AMD, as well as the impact of AMD and the effect of treatment on QoL. A total of 28 articles were selected.

Results: AMD has been found to cause a severity-dependent decrement in QoL that is comparable to systemic diseases such as cancer, ischemic heart disease, and stroke. QoL impairment manifests as greater social dependence, difficulty with daily living, higher rates of clinical depression, increased risk of falls, premature admission to nursing homes, and suicide. The National Eye Institute Visual Functioning Questionnaire (NEI VFQ-25) is the most widely used eye disease-specific QoL instrument in AMD. It has been shown to correlate significantly with visual acuity (VA). QoL reflects aspects of AMD including psychological well-being, functional capacity, and the ability to perform patients' valued activities, which are not captured by a single, numerical VA score.

Conclusion: The literature shows that the adverse impact of AMD on QoL is comparable to serious systemic disease. Eye disease-specific instruments for measuring QoL, such as the NEI VFQ-25, have shown a significant correlation of QoL decrement with measures of disease severity, as well as significant QoL improvement with treatment. The NEI VFQ-25 and other validated instruments provide a wide-ranging assessment of vision-related functioning that is important to patients and complementary to VA measurement. We strongly recommend the incorporation of QoL assessment into routine clinical practice.

Keywords: wet age-related macular degeneration, quality of life assessment, patient-reported outcome, clinical practice, review, NEI VFQ-25

\section{Background Age-related macular degeneration and associated burden of illness}

Age-related macular degeneration (AMD) is a chronic, progressive eye disorder that mainly affects people over the age of 50. ${ }^{1}$ Clinically, there are two types of AMD.
Correspondence: Mitsuko Yuzawa Surugadai Nihon University Hospital, Nihon University School of Medicine, I-8-I3, Kandasurugadai, Chiyoda-Ku,

Tokyo, Japan I0I-8309

Tel +8| 033293 |7| I

Email yuzawa.mitsuko@nihon-u.ac.jp 
Atrophic or "dry" AMD, also known as geographic atrophy, is characterized by fatty deposits behind the retina (drusen), causing thinning and drying of the macula. Dry AMD accounts for about $80 \%$ of cases affecting both eyes, but it typically causes only mild loss of vision. ${ }^{1}$ Neovascular AMD, or "wet" AMD (wAMD), is caused by growth of new blood vessels (choroidal neovascularization). The relative proportions of wAMD and dry AMD cases are dependent on the way they are defined. For example, findings from the Hisayama prospective cohort study in Japan revealed a higher wAMD prevalence, which was threefold (0.67\%) that of dry AMD $(0.2 \%) .^{2}$ Moreover, wAMD is associated with rapidly deteriorating vision and accounts for $90 \%$ of cases of severe visual impairment due to AMD. ${ }^{3}$

In the US, the estimated prevalence of AMD (neovascular and/or geographic atrophy) is $1.47 \%$, affecting approximately 1.75 million individuals. ${ }^{4}$ The burden of illness of wAMD due to lost wages alone is about $\$ 5.4$ billion. Other costs, such as those associated with caregiving, injury and provision of other services, drive the economic burden even higher., ${ }^{5,6}$ In Japan, which has the oldest and longest living population in the world, 1.64 million people are affected by visual impairment, of whom $61 \%$ are older than 65 years. Notably, early AMD is highly prevalent $(12.7 \%)$ in those 50 years and older in the Japanese population, whereas late AMD is less prevalent $(0.87 \%) .{ }^{2}$ More recently, follow-up from the ongoing Hisayama study showed a 9-year cumulative incidence of $10 \%$ for early AMD and $1.4 \%$ for late $\mathrm{AMD}^{7}{ }^{7}$ Overall, the economic burden of visual impairment in Japan is substantial, costing about 8785 billion Yen (US $\$ 72.8$ billion), or $1.7 \%$ of gross domestic product. ${ }^{8}$ Given the high prevalence of early AMD among the elderly Japanese population, it is likely that a significant proportion of this economic burden is due to AMD. In Asia, AMD is a major cause of blindness among the elderly. A high prevalence of AMD has also been documented in Singapore $(27 \%$ in those over 60 years of age) and Taiwan (9.2\% and $1.9 \%$ for early and late AMD, respectively, in those 65 years and older). ${ }^{9,10}$

\section{The importance of quality of life assessment in wAMD}

With progressive deterioration of the macula, AMD patients experience a multitude of visual problems that significantly affect their mental health and quality of life (QoL). In this context, it is notable that the World Health Organization constitution describes health as a state of physical, mental, and social well-being, not merely the absence of disease or infirmity. Consequently, the measurement of health should encompass an assessment of mental and social well-being, which can be accomplished by using QoL measures. ${ }^{11}$ Moreover, as health care continues to evolve from a "disease-based" to a "patient-centered" model, the importance of evaluating the outcomes of health care from the patient perspective is now widely recognized. Thus, in addition to clinical measures, a number of instruments measure patient-reported outcomes that assess patients' QoL, functional status, and their experiences with care.

"QoL is an individual's perception of position in life in the context of the culture and value systems in which they live and in relation to their goals, expectations, standards and concerns."

From the WHO "Measuring Quality of Life"11

Given the growing importance of incorporating QoL assessments into medical practice in general, and given the increasing prevalence of AMD in today's aging population and the heavy burden of this disease, it is critical to understand the impact of AMD on QoL and to improve the measurement of QoL in the context of routine ophthalmologic practice. The purpose of this paper is to provide a summary of the available evidence on the impact of AMD on patients' QoL and the utility of QoL assessments in evaluating the impact of AMD and its treatment, and to make a recommendation for incorporating QoL assessment into clinical practice.

\section{Methods}

We conducted an open Internet search and a PubMed search to identify articles (full text only) relating to the effects of wAMD on QoL. The following search criteria were used for the PubMed search: (1) English language, (2) peer reviewed journals, (3) articles published between 2000 and 2012. Search terms included "age-related macular degeneration," "wet AMD," "neovascular AMD," "burden of illness," "cost," "cost effectiveness," "prevalence," "blindness," "quality of life," "health-related quality of life," "patient-reported outcome," "questionnaire," "visual impairment," "activities of daily living," and "functional assessment."

A similar range of search terms was used in the open Internet search to identify other sources of information on vision-related QoL measures, QoL-specific treatment outcomes, guidelines concerning the role of QoL assessment in the clinical care of patients with AMD, and the impact of AMD on QoL. The primary targets of this search were websites representing professional societies and international health care organizations concerned with QoL and wAMD.

Literature from the PubMed and open Internet searches were reviewed and salient information was extracted. 


\section{Results}

\section{Clinical measurement of AMD severity}

In clinical practice, the severity of AMD can be categorized based on Snellen visual acuity (VA) testing of the betterseeing eye: mild (20/20-20/40), moderate (20/50-20/100), severe $(20 / 200$ or worse), very severe (20/800 or worse) (Table 1). ${ }^{6}$ Clinical practice in Japan uses the decimal-point equivalent of the VA. Traditionally, it has been the practice to treat patients with late-stage wAMD in clinical settings. However, with the availability of better diagnosis and effective treatment options, patients are evaluated earlier and those with AMD or even pre-AMD symptoms may receive treatment. Severity of AMD alone is no longer a sufficient criterion for treatment decision making. The impact of the disease on the patient's QoL is also an important factor. For example, while severe or very severe disease in one eye is an established criterion for treatment, patients with disease in both eyes may be treated regardless of VA to maintain vision-related QoL.

\section{Impact of AMD on QoL}

Given its increasing importance in clinical treatment of wAMD, it is critical to gauge the magnitude of QoL decrement in wAMD. It has been documented that compared to individuals without AMD and with vision within the normal range, those who are visually impaired experience significantly reduced QoL, which manifests as greater social dependence, difficulty with daily living, higher rates of clinical depression, increased risk of falls, premature admission to nursing homes, and suicide. ${ }^{3}$ The poor QoL associated with AMD is greatly underestimated by clinicians and the general public. ${ }^{12}$ AMD patients suffer from a multitude of visual problems including reduced central VA, impaired color vision, decreased contrast sensitivity, and metamorphosia. ${ }^{1}$ Although peripheral vision is usually retained in AMD, the losses in these multiple parameters of vision can impair proficiency in performing most activities of daily living and can make it more difficult for people to lead independent lives. ${ }^{3}$ Central vision, in particular, is needed for activities such as reading, driving, and facial recognition. Even in early AMD there is blurring and distortion of central vision, which decreases functional capacity for these activities. ${ }^{12}$ It is the progressive deterioration of central vision that profoundly impacts disease-specific QoL.

Patients in the earliest stages of AMD experience distorted vision, which has a minimal impact on their QoL (Table 1). Patients with moderately severe disease in one eye often experience difficulty reading small print but retain fairly normal distance vision, thereby reducing the overall effect on QoL. On the other hand, those with both eyes affected experience a major reduction in QoL due to poor facial recognition, difficulty writing or reading small print, and compromised ability to perform activities of daily living. ${ }^{1,3,6}$ Patients with advanced or late-stage AMD experience very poor QoL due to severely affected central vision and reduced VA (Table 1). These patients may have difficulty distinguishing between

Table I Impact of wAMD on QoL, treatment, and goals based on clinical assessments of WAMD severity

\begin{tabular}{|c|c|c|}
\hline Clinical assessment $^{1}$ & QoL impact & Treatment goals \\
\hline $\begin{array}{l}\text { Mild } \\
\text { - VA*: 20/20-20/40 } \\
\text { - Some distortion } \\
\text { - Detectable using OCT, funduscopy }\end{array}$ & $\begin{array}{l}\text { - None to minimal impact on QoL; } \\
\text { patients feel something is slightly } \\
\text { wrong with their vision }\end{array}$ & $\begin{array}{l}\text { - Eligible for treatment } \\
\text { - Stabilization or improvement } \\
\text { of VA possible }\end{array}$ \\
\hline $\begin{array}{l}\text { Moderate to severe } \\
\text { - VA*: 20/50-20/200 } \\
\text { - One eye: distance vision is still fairly good, since the other } \\
\text { better eye helps with overall vision; however, still some } \\
\text { difficulties (eg, writing or reading small print) } \\
\text { - Both eyes: difficulty in daily activity, poor facial recognition, } \\
\text { cannot read and write small print, letters, or bank statements } \\
\text { - Central dark spots (size is important; eg, } \\
\text { absolute central scotoma) }\end{array}$ & $\begin{array}{l}\text { - One eye: QoL not greatly } \\
\text { affected, but some patients } \\
\text { feel anxiety for the other eye } \\
\text { possibly developing AMD } \\
\text { - Both eyes: QoL greatly affected }\end{array}$ & $\begin{array}{l}\text { - Eligible for treatment } \\
\text { - Improvement of VA and maintenance } \\
\text { of improved VA, maintaining QoL } \\
\text { - For one eye, elimination of a central } \\
\text { dark spot possible, and also may } \\
\text { improve mental health by reducing } \\
\text { anxiety for the other eye possibly } \\
\text { developing AMD }\end{array}$ \\
\hline $\begin{array}{l}\text { Very severe } \\
\text { - VA*: }<20 / 200 \\
\text { - Darkness; cannot see anything in center vision from } \\
\text { affected eye, difficulty driving and distinguishing color }\end{array}$ & - Severe & $\begin{array}{l}\text { - Not eligible for those with a scar; } \\
\text { clear atrophy of cells or clear eye } \\
\text { damage (disease too advanced to see } \\
\text { treatment effect) }\end{array}$ \\
\hline
\end{tabular}

Note: *Snellen VA can be converted to decimal VA by dividing the numerator by the denominator (eg, 20/20=1;20/40=0.5).

Abbreviations: wAMD, wet age-related macular degeneration; QoL, quality of life; VA, visual acuity; OCT, optical coherence tomography; AMD, age-related macular degeneration. 
certain colors. Thus, AMD has been found to cause a severitydependent decrement in QoL ranging from 17\% to $63 \%$, which is comparable to systemic diseases such as cancer, ischemic heart disease, and stroke (Figure 1). ${ }^{6}$ The impact of QoL is also an important part of patient management, enhancing the clinical assessment based on improvement or stabilization of VA. Table 1 summarizes the treatment goals for AMD, in conjunction with the corresponding clinical assessments and QoL impact by disease severity.

\section{Value of QoL measures in patient assessments and current use in WAMD clinical research}

Change in VA is the standard measure in clinical practice as well as in trial settings for evaluating treatment response in AMD. However, VA or other measures of disease severity (eg, optical coherence tomography) alone do not take into account the impact of all aspects of the disease, including such manifestations as central dark spots, vision-related functioning, the capacity to engage in valued activities, or the psychological effect of changes in these parameters (ie, the patient's actual QoL). ${ }^{13-15}$ From the patient's perspective, improvement in psychological well-being and the ability to perform activities of daily living that are dependent on visual function may be as, or more, important than the clinical assessment of VA itself. ${ }^{13,15}$ The importance of recognizing disease-specific QoL in AMD is manifold. First, it justifies the need to prevent disease progression with therapeutic interventions. Second, it provides a means of measuring the impact of treatment on what matters most patients' ability to function and enjoy life. Third, it encourages

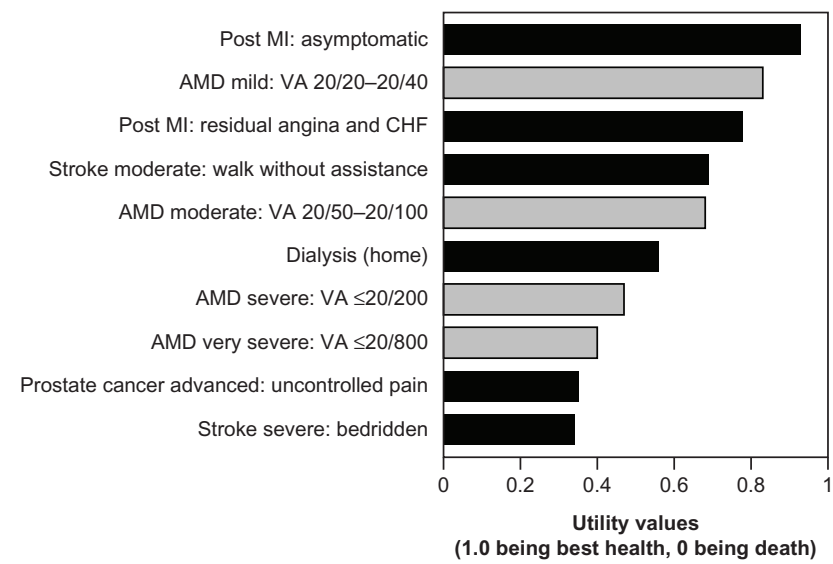

Figure I Impact of AMD on QoL as presented by utility values corresponding to VA in the better seeing eye, compared with utility values for other disease states. Note: This figure is created from a table in an article by Brown et al, entitled, "The burden of age-related macular degeneration: a value-based medicine analysis" in Trans Am Ophthalmol Soc. 2005; 103:173-184. ${ }^{6}$ Adapted with permission from the American Ophthalmological Society.

Abbreviations: AMD, age-related macular degeneration; QoL, quality of life; VA, visual acuity; MI, myocardial infarction; CHF, congestive heart failure. the development of low-vision aids and other devices to help patients with activities of daily living by providing a means of quantifying the benefits, in terms of improved functional capacity, of interventions that do not alter unaided VA. ${ }^{12}$

Although VA and QoL are clearly correlated, the latter is what matters most to the patient and is the ultimate measure of the need for, and success of, treatment. ${ }^{16}$ Fortunately, there are a number of disease-specific QoL instruments that have been validated in the wAMD population that can be used to directly measure their QoL. The National Eye Institute Visual Functioning questionnaire (NEI VFQ-25), the Daily Living Tasks Dependent on Vision questionnaire (DLTV), the Activities of Daily Vision Scale, and the Visual Function Index are prominent examples of vision-specific QoL questionnaires designed to assess respondents' health status, functional status, visual function, and well-being. ${ }^{3}$

The NEI VFQ-25, a 25-item questionnaire, is one of the most widely used of these instruments (Table 2). ${ }^{17}$ Several of the subscales of this instrument have been used as prespecified endpoints in clinical trials: near vision (items 5-7), distance vision (items 8, 9, 14), dependency (items 20, 23, 24), and mental health (items $3,21,22,25$ ). In a noninterventional study of wAMD patients, Orr et $\mathrm{al}^{18}$ used the NEI VFQ-25 total composite score and subscale scores for near activity, distance activity, and vision-specific dependency to correlate with clinical assessment (best corrected VA) and to associate with patient-reported outcomes (restricted activity days), a measure of QoL. Their results showed that the NEI VFQ-25 composite and subscale scores were significantly correlated with best corrected VA of the better-seeing eye $(\mathrm{r}=-0.48$ to -0.54 ; all $P<0.0001)$ and with restricted activity days (r $=-0.52$ to -0.55 ; all $P<0.0001$ ). Patients with fewer restricted activity days over a 3-month period had a significantly higher NEI VFQ-25 score (eg, patients with 0-1 days of restricted activity had a mean NEI VFQ-25 total composite score of 79.8 compared with a score of 43.5 for those with $>9$ restricted activity days $[P<0.001])$. Subscale scores showed similar results to total composite scores (Figure 2). In the Age-Related Eye Disease Study, ${ }^{19} \mathrm{NEI}$ VFQ-25 was administered to AMD patients twice within a 1- to 4-year interval to assess the impact of disease progression and loss of VA on health-related QoL. The NEI VFQ25 composite score was significantly responsive to disease progression $(t=14 ; P<0.001)$ and to loss of VA $(t=16$; $P<0.001) .{ }^{20}$ Subscale scores - including near activities, distance activities, dependency, and mental health scores were also significantly responsive, though the effect sizes were smaller than those for the overall score. These studies 
Table 2 Item analysis of NEI VFQ-25

\begin{tabular}{|c|c|c|c|}
\hline $\mathbf{N}$ & Item & Subscale & $\begin{array}{l}\text { Measure of } \\
\text { response }\end{array}$ \\
\hline 2 & General vision & General vision & $\begin{array}{l}\text { Quality (Excellent/ } \\
\text { Good/Fair/Poor/ } \\
\text { very poor/completely } \\
\text { blind) }\end{array}$ \\
\hline 3 & Worry about eyesight & Mental health & Frequency $^{\mathrm{a}}$ \\
\hline 5 & Reading normal newsprint & Near vision & Difficulty ${ }^{b}$ \\
\hline 6 & Seeing well up close & Near vision & Difficulty ${ }^{\mathrm{b}}$ \\
\hline 7 & $\begin{array}{l}\text { Finding objects on } \\
\text { crowded shelf }\end{array}$ & Near vision & Difficulty ${ }^{b}$ \\
\hline 8 & Street signs & Distance vision & Difficulty ${ }^{b}$ \\
\hline 9 & Going downstairs at night & Distance vision & Difficulty ${ }^{b}$ \\
\hline $\mathrm{II}$ & Seeing how people react & Social function & Difficulty ${ }^{b}$ \\
\hline 12 & Matching clothes & Color vision & Difficulty ${ }^{b}$ \\
\hline 13 & Visiting others & Social function & Difficulty ${ }^{b}$ \\
\hline 14 & Going out to movies/plays & Distance vision & Difficulty ${ }^{b}$ \\
\hline 15 & Driving in daylight & Driving & $\begin{array}{l}\text { Difficulty [no/a little/ } \\
\text { moderate/extreme] }\end{array}$ \\
\hline 16 & Driving in difficult conditions & Driving & Difficulty ${ }^{b}$ \\
\hline 17 & Accomplish less & Role limitation & Frequency $^{\mathrm{a}}$ \\
\hline 18 & Limited endurance & Role limitation & Frequency $^{a}$ \\
\hline 20 & Stay home most of the time & Dependency & Agreement $^{c}$ \\
\hline 21 & Frustrated & Mental health & Agreement $^{c}$ \\
\hline 22 & No control & Mental health & Agreement $^{c}$ \\
\hline 23 & $\begin{array}{l}\text { Rely too much on others' } \\
\text { words }\end{array}$ & Dependency & Agreement $^{c}$ \\
\hline 24 & $\begin{array}{l}\text { Needed much help from } \\
\text { others }\end{array}$ & Dependency & Agreement $^{c}$ \\
\hline 25 & Embarrassment & Mental health & Agreement $^{c}$ \\
\hline
\end{tabular}

Notes: aNone of the time/a little of the time/some of the time/most of the time/ all of the time. 'No difficulty/a little difficulty/moderate difficulty/extreme difficulty/ stopped doing because of eyesight/stopped doing for other reasons or not interested in doing. 'Definitely true/mostly true/not sure/mostly false/definitely false.

This table is modified from an article by Marella et al, entitled, "The psychometric validity of the NEI VFQ-25 for use in a low-vision population" in Invest Ophthalmol Vis Sci. 20 I0;5 I (6):2878-2884 and republished with permission of the copyright holder, the Association for Research in Vision and Ophthalmology. ${ }^{17}$

Abbreviation: NEI VFQ-25, National Eye Institute Visual Functioning Questionnaire 25-items.

demonstrate that the NEI VFQ-25 is a valid measure of vision-related QoL in AMD.

Another example of a vision-specific instrument is DLTV, a 24-item questionnaire that assesses tasks dependent on distance and near vision, depth and contrast perception, light and dark adaptation, and visual fields. ${ }^{22}$ The construct validity of this instrument was demonstrated by showing a statistically significant correlation of the DLTV total and domain scores while using, versus not using, regular help from caregiving $(P<0.0001){ }^{23}$ For example, patients receiving regular help from caregiving had a mean DLTV total score of $49.8 \pm 20.4$, while those not requiring regular help had a score of $79.0 \pm 18.1(P<0.0001)$.

Although vision-specific instruments provide disease-specific QoL information, generic QoL instruments (ie, do not target

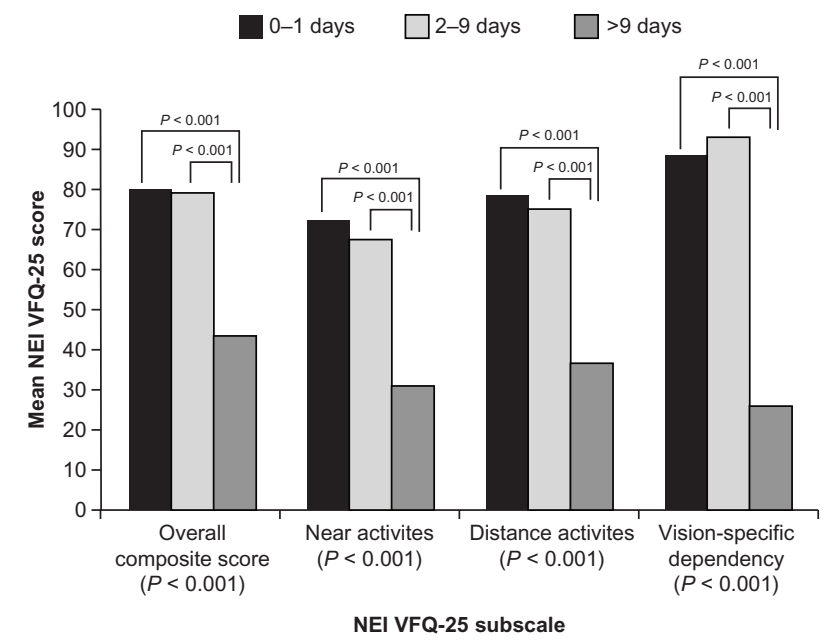

Figure 2 NEI VFQ-25 composite and subscale scores by number of patient-reported restricted activity days due to AMD over a 3-month period.

Notes: Analysis of covariance model adjusted for age and sex. This figure is reproduced from an article by Orr et al, entitled, "Validation of the National Eye Institute Visual Function Questionnaire-25 (NEI VFQ-25) in age-related macular degeneration" in Invest Ophthalmol Vis Sci. 20I I;52(6):3354-3359 and republished with permission of the copyright holder, the Association for Research in Vision and Ophthalmology. ${ }^{18}$

Abbreviations: NEI VFQ-25, National Eye Institute Visual Functioning Questionnaire 25-items; AMD, age-related macular degeneration.

a specific age, disease, or treatment group) such as the Short Form-12 or Short Form-36, have also been used to assess QoL in patients with AMD. These instruments provide functional health and well-being scores and a preference-based health utility index, as well as psychometrically based physical and mental health summary measures. ${ }^{24}$ However, general QoL instruments have been shown only to clearly differentiate between different ophthalmic disease states, but not to be responsive to disease progression or changes in VA in wAMD. ${ }^{24}$

Most clinical studies depend on quantitative outcome measures, but the value of therapy to the patient and caregiver depends more directly on improvement in QoL. Pharmacotherapy with pegaptanib, ranibizumab, and intravitreal aflibercept have all demonstrated significant improvements in QoL composite score on the NEI VFQ-25, indicating overall improvement in the respondent's visual health and related functional capacities. ${ }^{13,14,25,26}$ Other benefits, such as a reduction in the incidence of depression or a decrease in injuries due to falls (a major hazard of vision loss), may also be reflected in improved QoL scores. ${ }^{3,12}$ More specifically, a reduction in dependency is manifested as improvements in daily activities such as reading, cooking, identifying objects on a crowded shelf, watching television, shopping, doing hobbies such as sewing and fixing things, and being able to walk across streets without assistance (Table 3). ${ }^{13,14,25,26}$ Additionally, improvements in QoL composite score were documented, indicating overall improvement in the respon- 
Table 3 QoL assessment in clinical trials of antivascular endothelial growth factor therapy for wAMD

\begin{tabular}{|c|c|c|}
\hline Study & Treatment & QoL endpoints \\
\hline $\begin{array}{l}\text { VISION. }^{26} \\
\text { A prospective, randomized, } \\
\text { double-blind, multicenter, } \\
\text { dose-ranging study }\end{array}$ & $\begin{array}{l}\text { Pegaptanib }(0.3 \mathrm{mg}, \mathrm{I} \mathrm{mg} \text {, } \\
\text { and } 3 \mathrm{mg}) \text { versus standard } \\
\text { of care }\end{array}$ & $\begin{array}{l}\text { NEI VFQ-25: near and distance } \\
\text { vision, role limitations, and } \\
\text { dependency score changes from } \\
\text { baseline to endpoint }\end{array}$ \\
\hline $\begin{array}{l}\text { MARINA. }{ }^{14} \\
\text { A randomized, double- } \\
\text { masked, dose-ranging } \\
\text { study }\end{array}$ & $\begin{array}{l}\text { Ranibizumab ( } 0.3 \mathrm{mg} \\
\text { or } 0.5 \mathrm{mg} \text { ) versus sham }\end{array}$ & $\begin{array}{l}\text { NEI VFQ-25 overall composite } \\
\text { score and subscale score } \\
\text { changes from baseline to } \\
\text { endpoint }\end{array}$ \\
\hline
\end{tabular}

Outcome*

\section{ANCHOR. ${ }^{13}$}

A randomized, multicenter, double-masked, doseranging study
Ranibizumab (0.3 mg or $0.5 \mathrm{mg}$ ) plus sham verteporfin versus sham injections plus active verteporfin
NEI VFQ-25 overall composite score and subscale score changes from baseline to endpoint

At week 54, distance vision and role limitations domains were significantly improved in pegaptanib versus usual care, with LS differences of 4-6 points overall, and 6-8 points for $3 \mathrm{mg}$ dose. At 12 months, significant improvement in composite scores of 5-6 points was noted with both doses compared to a decline of 3 points with sham. Significant improvement of $>4$ points was observed for six of 12 subscales at the $0.5 \mathrm{mg}$ dose. At 24 months, the largest improvements ( $>8$ points) were seen in the subscales near activities, general vision, and mental health.

At 12 months, improvement in composite scores was noted with both $0.3 \mathrm{mg}$ and $0.5 \mathrm{mg}$ doses (5.9 and 8.1 points, respectively), which was significantly greater compared to verteporfin photodynamic therapy $(P<0.0 \mathrm{I}$ and $P<0.00 \mathrm{I})$. At 24 months, greater improvement was observed in ranibizumab-treated patients on most subscales, including near and distance activities and dependency.

MARINA and ANCHOR. ${ }^{21}$

Ranibizumab ( 0.3 or 0.5 mg) versus sham Data were analyzed separately for MARINA and ANCHOR and treatment groups were pooled within each trial

Intravitreal aflibercept $(0.5 \mathrm{mg}$ q4w; 2 mg q4w; 2 mg q8w after 3 loading doses $q 4 w$ ) versus ranibizumab $0.5 \mathrm{mg}$ $q 4 w$. Data were integrated from two highly similarly designed trials
Regression models were used to assess change from baseline to 12 months in NEI VFQ-25 composite and subscale scores. Changes in VA at 12 months (>I5 letters gained, < I 5 letters lost, $>$ I 5 letters gained or lost) Regression models were used to assess change from baseline to 12 months in NEI VFQ-25 composite and subscale scores. Intravitreal aflibercept outcomes were compared to ranibizumab outcomes

Note: *Improvement of four points or more in NEI VFQ-25 scores represents a clinically meaningful change in visual acuity. ${ }^{21}$

Abbreviations: QoL, quality of life; wAMD, wet age-related macular degeneration; VISION, Vascular endothelial growth factor Inhibition Study In Ocular Neovascularization; NEI VFQ-25, National Eye Institute Visual Functioning Questionnaire 25-items; LS, least squares; MARINA, Minimally classic/occult trial of the Antivascular endothelial growth factor antibody Ranibizumab In the treatment of Neovascular Age-related macular degeneration; ANCHOR, Antivascular endothelial growth factor antibody for the treatment of predominantly classic CHoroidal Neovascularization in age-related macular degeneration; VIEW, Vascular endothelial growth factor trap-eye; Investigation of Efficacy and safety in Wet age-related macular degeneration; q4w, every 4 weeks; q8w, every 8 weeks; VA, visual acuity.

dents' mental and physical health and reduction in injuries due to falls, a major hazard of vision loss.

\section{Discussion}

The literature on QoL in AMD, as briefly reviewed here, has established that the impact of WAMD on QoL is severitydependent and quantitatively similar to that for other major diseases of the elderly. Second, there are several visionspecific instruments for measuring QoL that have been proven for reliability and validity, and which are superior to general health-related QoL instruments in this population. Third, vision-specific QoL instruments, such as the NEI VFQ-25, have been shown to be sensitive to disease progression, as measured by the worsening of VA. Most recently, and of great clinical importance, clinical trials of antivascular endothelial growth factor treatments of wAMD using the NEI VFQ-25 have demonstrated substantial improvements in QoL that parallel the improvement in VA.

Despite the demonstrated vision-related QoL benefits of treatment observed in research settings and the recurrent recognition by clinical researchers that these benefits are the most meaningful to patients, the measurement of visionrelated QoL is quite limited in real-world clinical practice. Integrating the assessment of QoL into clinical practice 
may increase patients' motivation to adhere to treatment by providing them with objective feedback on the real-life improvements they have attained. It would also provide the clinician with an additional common language for discussing treatment progress with the patient. In addition, routine QoL assessment will generate outcome data that may help demonstrate the cost effectiveness of this expensive treatment to key stakeholders, including physicians, payers, and regulatory agencies.

Perhaps the major barrier to the implementation of a QoL assessment is insufficient time on the part of the attending ophthalmologist to administer the instrument routinely during clinic visits. For example, in our experience, the intervieweradministered version has typically taken approximately 10 to 15 minutes to complete per patient. Some of the difficulty in accomplishing the task more efficiently is due to patient factors, such as poor VA and/or cognitive impairment. Of equal importance, however, is the extensiveness of the instruments themselves, which were designed primarily for research purposes. With respect to the NEI VFQ-25, for example, it is inevitably time consuming to administer a 25-item questionnaire.

Based on the literature reviewed here, it is clear that some NEI VFQ-25 subscales and their corresponding items have a greater impact on vision-related QoL assessment in AMD patients than others. In recognizing the busy schedules of clinicians, whose primary focus is to assess changes in VA, we recommend that the original NEI VFQ-25 be shortened to include only those items that are most relevant to assessing QoL in wAMD patients in the clinical setting. By shortening the administration time and also making scoring and interpretation easier, an abridged version of the NEI VFQ-25 would be more readily adopted by practicing clinicians. However, further research evaluating the reliability and validity of such an instrument would be required.

Another barrier to adopting a QoL assessment may be that clinicians are not aware that some of the instruments described above have been validated in many regions of the world. ${ }^{17}$ With regard to eye disease-specific QoL instruments, the NEI VFQ-25 has been psychometrically validated in many languages with minor amendments made to the original instrument in order to accommodate wider usage. ${ }^{15,27,28}$ For example, there is a Japanese version of the NEI VFQ-25 that was modified to substitute items in the near and distance vision subscales with those that are more relevant to Japanese patients. ${ }^{15}$ Such amendments minimize missing or inaccurate data and thereby increase measurement precision.
Adoption of a QoL assessment in clinical practice might increase if it were recommended in clinical guidelines for the evaluation and management of wAMD. For example, QoL assessment using a validated questionnaire is specifically discussed and recommended in the Japanese guidelines for allergic rhinitis. ${ }^{29}$ Treatment for allergic rhinitis is aimed at alleviating symptoms and eliminating difficulties experienced in everyday life, hence resulting in an improvement in QoL. Improvement in QoL was included in the guidelines because allergic rhinitis is manageable when treated, although it resists cure. ${ }^{30}$

\section{Conclusion}

wAMD accounts for $90 \%$ of cases of severe visual impairment due to AMD, and epidemiological data clearly show that this form of visual impairment typically causes a significant reduction in QoL and substantial societal burden. Decrements in QoL associated with wAMD can be easily and accurately measured, and research has clearly demonstrated the value of QoL assessment for understanding the natural history of wAMD and its response to treatment. QoL measures additional aspects of the disease, such as the psychological well-being and daily functioning of patients, that cannot be captured by a single clinical measurement of disease severity. However, despite their widespread use in epidemiologic research and clinical trial settings, eye disease-specific QoL measures are seldom used in routine clinical assessment of AMD patients, in part because of barriers to implementation. The present review indicates that efforts to overcome these barriers are warranted. The authors recommend the development of a validated shortened version of the NEI VFQ-25, tailored specifically to wAMD, for use in QoL assessment in the routine clinical care of wAMD patients.

\section{Acknowledgments}

The authors wish to acknowledge the contribution of Kim Ullerup Wittrup-Jensen, Kenji Adachi, and Keigo Kudo of Bayer for their insightful comments during the manuscript preparation and discussion. Medical writing assistance was provided by Bagi Ravishankar, PhD, and Bill Kadish, MD, of PAREXEL, and was funded by Bayer HealthCare, LLC.

All authors contributed important intellectual content, including discussions on the manuscript concept and outline, interpretation of the literature review results, clinical insights, and critical revision of all drafts of the manuscript. All authors approved the final draft for submission and take full responsibility for the contents of this article. 


\section{Disclosure}

MY and KF have received fees for consulting from Bayer Yakuhin, Ltd. ET and EW are salaried employees of Bayer Yakuhin, Ltd. The authors report no other conflicts of interest in this work.

\section{References}

1. Jager RD, Mieler WF, Miller JW. Age-related macular degeneration. N Engl J Med. 2008;358(24):2606-2617.

2. Oshima Y, Ishibashi T, Murata T, Tahara Y, Kiyohara Y, Kubota T. Prevalence of age related maculopathy in a representative Japanese population: the Hisayama study. Br J Ophthalmol. 2001;85(10): 1153-1157.

3. Mitchell J, Bradley C. Quality of life in age-related macular degeneration: a review of the literature. Health Qual Life Outcomes. 2006;4:97.

4. Friedman DS, O’Colmain BJ, Muñoz B, et al; for Eye Disease Prevalence Research Group. Prevalence of age-related macular degeneration in the United States. Arch Ophthalmol. 2004;122(4):564-572.

5. AMD Alliance International. The Global Economic Cost of Visual Impairment: Summary Report. 2010. Available from: http://www.amdalliance.org/ amdalliance/AMDAlliance/cost-of-blindness. Accessed May 20, 2013.

6. Brown GC, Brown MM, Sharma S, et al. The burden of age-related macular degeneration: a value-based medicine analysis. Trans Am Ophthalmol Soc. 2005;103:173-184; discussion 184-186.

7. Yasuda M, Kiyohara Y, Hata Y, et al. Nine-year incidence and risk factors for age-related macular degeneration in a defined Japanese population the Hisayama study. Ophthalmology. 2009;116(11):2135-2140.

8. Roberts CB, Hiratsuka Y, Yamada M, et al. Economic cost of visual impairment in Japan. Arch Ophthalmol. 2010;128(6):766-771.

9. Woo JH, Au Eong KG. Don't lose sight of age-related macular degeneration: the need for increased awareness in Singapore. Singapore Med $J$. 2008;49(11):850-853.

10. Chen SJ, Cheng CY, Peng KL, et al. Prevalence and associated risk factors of age-related macular degeneration in an elderly Chinese population in Taiwan: the Shihpai Eye Study. Invest Ophthalmol Vis Sci. 2008;49(7):3126-3133.

11. World Health Organization. WHOQOL: Measuring quality of life. 1997. Available from: http://www.who.int/mental_health/media/68. pdf. Accessed May 20, 2013.

12. Stein JD, Brown MM, Brown GC, Hollands H, Sharma S. Quality of life with macular degeneration: perceptions of patients, clinicians, and community members. Br J Ophthalmol. 2003;87(1):8-12.

13. Bressler NM, Chang TS, Fine JT, Dolan CM, Ward J; for Anti-VEGF Antibody for the Treatment of Predominantly Classic Choroidal Neovascularization in Age-Related Macular Degeneration (ANCHOR) Research Group. Improved vision-related function after ranibizumab vs photodynamic therapy: a randomized clinical trial. Arch Ophthalmol. 2009;127(1):13-21.

14. Chang TS, Bressler NM, Fine JT, et al. Improved vision-related function after ranibizumab treatment of neovascular age-related macular degeneration: results of a randomized clinical trial. Arch Ophthalmol. 2007;125(11):1460-1469.

15. Suzukamo Y, Oshika T, Yuzawa M, et al. Psychometric properties of the 25-item National Eye Institute Visual Function Questionnaire (NEI VFQ-25), Japanese version. Health Qual Life Outcomes. 2005;3:65.

Clinical Ophthalmology

\section{Publish your work in this journal}

Clinical Ophthalmology is an international, peer-reviewed journal covering all subspecialties within ophthalmology. Key topics include: Optometry; Visual science; Pharmacology and drug therapy in eye diseases; Basic Sciences; Primary and Secondary eye care; Patient Safety and Quality of Care Improvements. This journal is indexed on Submit your manuscript here: http://www.dovepress.com/clinical-ophthalmology-journal
16. Miskala PH, Bass EB, Bressler NM, et al; for Submacular Surgery Trials (SST) Research Group. Surgery for subfoveal choroidal neovascularization in age-related macular degeneration: quality-of-life findings: SST report no 12. Ophthalmology. 2004;111(11):1981-1992.

17. Marella M, Pesudovs K, Keeffe JE, O'Connor PM, Rees G, Lamoureux EL. The psychometric validity of the NEI VFQ-25 for use in a low-vision population. Invest Ophthalmol Vis Sci. 2010;51(6): $2878-2884$.

18. Orr P, Rentz AM, Margolis MK, et al. Validation of the National Eye Institute Visual Function Questionnaire-25 (NEI VFQ-25) in age-related macular degeneration. Invest Ophthalmol Vis Sci. 2011;52(6):3354-3359.

19. Age-Related Eye Disease Study Research Group. The Age-Related Eye Disease Study (AREDS): design implications. AREDS report no 1. Control Clin Trials. 1999;20(6):573-600.

20. Lindblad AS, Clemons TE. Responsiveness of the National Eye Institute Visual Function Questionnaire to progression to advanced age-related macular degeneration, vision loss, and lens opacity: AREDS Report no 14. Arch Ophthalmol. 2005;123(9):1207-1214.

21. Suñer IJ, Kokame GT, Yu E, Ward J, Dolan C, Bressler NM. Responsiveness of NEI VFQ-25 to changes in visual acuity in neovascular AMD: validation studies from two phase 3 clinical trials. Invest Ophthalmol Vis Sci. 2009;50(8):3629-3635.

22. McClure ME, Hart PM, Jackson AJ, Stevenson MR, Chakravarthy U. Macular degeneration: do conventional measurements of impaired visual function equate with visual disability? $\mathrm{Br} J$ Ophthalmol. 2000;84(3):244-250.

23. Schmier JK, Halpern MT, Covert D. Validation of the Daily Living Tasks Dependent on Vision (DLTV) questionnaire in a U S population with age-related macular degeneration. Ophthalmic Epidemiol. 2006;13(2):137-143.

24. Globe DR, Levin S, Chang TS, Mackenzie PJ, Azen S. Validity of the SF-12 quality of life instrument in patients with retinal diseases. Ophthalmology. 2002;109(10):1793-1798.

25. Heier JS, Brown DM, Chong V, et al; for VIEW 1 and VIEW 2 Study Groups. Intravitreal aflibercept (VEGF trap-eye) in wet age-related macular degeneration. Ophthalmology. 2012;119(12): 2537-2548.

26. Leys A, Zlateva G, Shah SN, Patel M. Quality of life in patients with age-related macular degeneration: results from the VISION study. Eye (Lond). 2008;22(6):792-798.

27. Chan CW, Wong D, Lam CL, McGhee S, Lai WW. Development of a Chinese version of the National Eye Institute Visual Function Questionnaire (CHI-VFQ-25) as a tool to study patients with eye diseases in Hong Kong. Br J Ophthalmol. 2009;93(11):1431-1436.

28. Labiris G, Katsanos A, Fanariotis M, et al. Psychometric properties of the Greek version of the NEI-VFQ 25. BMC Ophthalmol. 2008;8:4.

29. Okuda M, Ohkubo K, Goto M, et al. Comparative study of two Japanese rhinoconjunctivitis quality-of-life questionnaires. Acta Otolaryngol. 2005;125(7):736-744.

30. Okubo K, Kurono Y, Fujieda S, et al; for Japanese Society of Allergology. Japanese guideline for allergic rhinitis. Allergol Int. 2011;60(2):171-189.

PubMed Central and CAS, and is the official journal of The Society of Clinical Ophthalmology (SCO). The manuscript management system is completely online and includes a very quick and fair peer-review system, which is all easy to use. Visit http://www.dovepress.com/ testimonials.php to read real quotes from published authors. 